\section{G300(P) PATIENTS WITH MUCOPOLYSACCHARIDOSIS AND INFANTILE ONSET POME DISEASE ARE AT INCREASED RISK OF RESPIRATORY MORBIDITY AND OBSTRUCTIVE SLEEP APNOEA}

${ }^{1}$ Aditi Sinha, ${ }^{2}$ Alex Broomfield, ${ }^{2}$ Simon Jones, ${ }^{1}$ Stuart Wilkinson, ${ }^{1}$ Omendra Narayan. ${ }^{1}$ Department of Paediatric Respiratory Medicine, Royal Manchester Children's Hospital, Manchester, UK; ${ }^{2}$ Department of Paediatric Metabolic Medicine, Royal Manchester Children's Hospital, Manchester, UK

\subsection{6/archdischild-2020-rcpch.261}

Introduction Respiratory disease complicates the management of several inherited metabolic diseases, increasing morbidity, mortality. All patients should therefore undergo detailed diagnostic work-up \& regular monitoring via a multi-disciplinary approach.

Aims To gather epidemiological data on the respiratory manifestations of children with inherited metabolic diseases at a quaternary metabolic center.

Method Using a database of all patients with inherited metabolic disease (total patients: 1022), those with input from respiratory/ENT services were identified. Using in-house IT systems, data was collected including patient demographics, diagnosis of airway pathology, management including use of invasive (IV) and non-invasive ventilation (NIV) and follow up data.

Results In total 76 patients were identified as requiring input from respiratory/ENT services. Table 1 outlines the main results, detailing the number of patients within each group of inherited metabolic disease, number requiring NIV or IV, mean age at onset of respiratory support and the number of patients with follow up arranged. 26\% (17/65) of patients with Mucopolysaccharidosis (MPS) had a diagnosis of obstructive sleep apnoea (OSA).

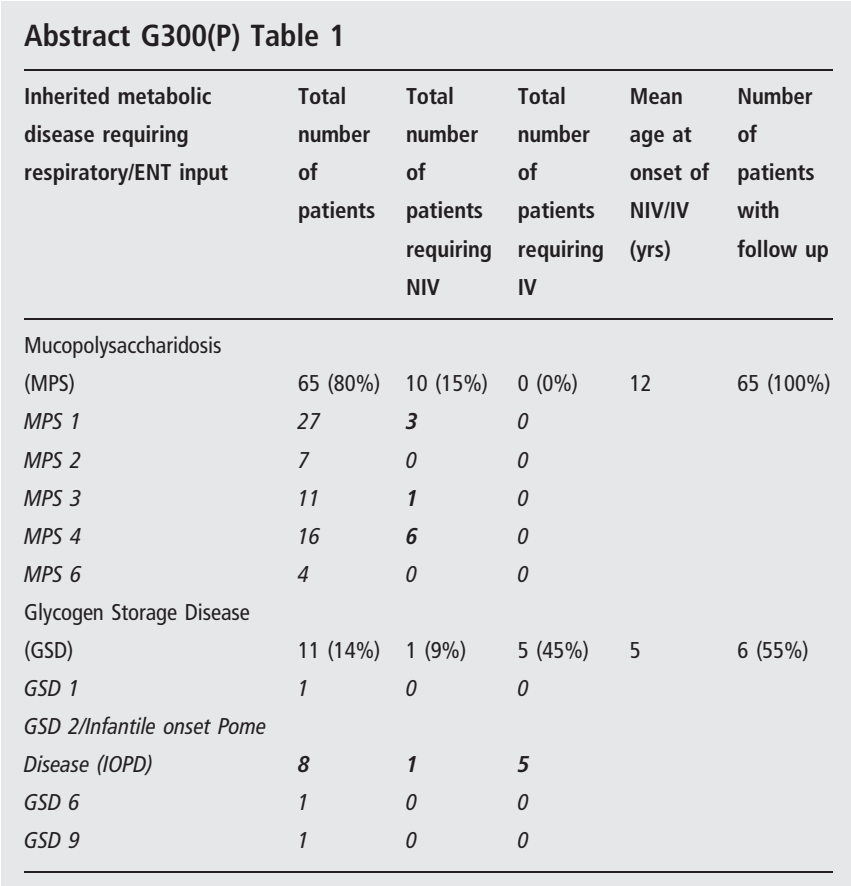

Conclusion Patients with MPS and IOPD disease require respiratory support in many cases. Those with MPS are at risk of OSA due to glycosaminoglycan deposition and tortuosity of airways. Alveolar hypoventilation due to respiratory muscle dysfunction occurs in those with IOPD leading to type 2 respiratory failure. Recognition of these manifestations is essential, as early intervention has a significant impact in slowing the decline of previously fatal complications improving the quality of life for these patients.

\section{G301(P) DIAGNOSING HEREDITARY HAEMORRHAGIC TELANGIECTASIA IN CHILDREN: A SERVICE EVALUATION PROJECT}

${ }^{1}$ ER Anderson, ${ }^{2} \mathrm{MG}$ Semple. 'Liverpool Centre for Genomic Medicine, Liverpool Women's Hospital, Liverpool, UK; ${ }^{2}$ Faculty of Health and Life Sciences, University of Liverpool, Liverpool, UK

\subsection{6/archdischild-2020-rcpch.262}

Background Hereditary haemorrhagic telangiectasia (HHT), also known as Osler-Weber-Rendu syndrome, is an autosomal dominant disorder characterised by telangiectasia, epistaxis, and visceral arterio-venous malformations (AVMs). The value of Curaçao diagnostic criteria may be limited in young children due to age-related penetrance of clinical features. Treatment of pulmonary and cerebral AVMs can improve long-term morbidity and mortality. Prophylactic antibiotics are recommended in specific circumstances.

Aims

- To assess how many children receive a molecular diagnosis of HHT in our region.

- To review the utility of the Curaçao criteria in children with molecularly confirmed HHT.

Design A retrospective electronic case-note review of all patients assessed in a regional Clinical Genetics service between January 2012 and December 2017 was undertaken. Potential cases were identified by searching clinic letters for keywords ('HHT' or 'telang*') with matched review of molecular analysis.

Results 23 children (age $\leq 16$ years) were identified.

Seven children $(3-15$ years) were referred with a clinical suspicion of HHT. Five of these children subsequently underwent genetic testing, with a molecular diagnosis of HHT confirmed in 2 (40\%). The Curaçao score for the 2 children with HHT was 3, compared to 2 in all those without a molecular diagnosis.

Sixteen children (6 days - 16 years) were referred due to a parent having a molecular diagnosis of HHT. Of these, fifteen children underwent genetic testing, with the familial variant present in 8 (53\%). The mean Curaçao scores for children with and without a molecular diagnosis were 2.1 (1-3) and $1.3(1-2)$ respectively $(p=0.06)$.

A further 13 children were identified to have a first degree relative with confirmed HHT. Half of these children would be expected to have a molecular diagnosis of HHT.

Conclusion In total, 10 children had a confirmed molecular diagnosis of HHT. As others have reported, a low Curaçao score is unreliable for excluding HHT in children. HHT should be considered in any child with AVMs, severe epistaxis or unexplained hypoxia. All children with a first degree relative with molecularly confirmed HHT should be offered genetic testing. Children with a molecular diagnosis or high clinical suspicion of HHT should be referred to a paediatrician with a special interest in HHT. 